\title{
Management of interstitial cystitis/bladder pain syndrome
}

\author{
Ashley Cox, MD, MSc, FRCSC
}

Department of Urology, Dalhousie University, Halifax, NS, Canada

Cite as: Can Urol Assoc J 2018;12(6Suppl3):S157-60. http://dx.doi.org/10.5489/cuaj.5324

(three-day voiding diary), survey scores (examples given in Dr. Carr's section on evaluation), urinalysis, and culture results. This objective information can then be reviewed before your interview to save time for the physical exam and treatment plan.

Lastly, to help make the most of the initial visit and subInterstitial cystitis/bladder pain syndrome (IC/BPS) is a clinical diagnosis based primarily on chronic symptoms of pain perceived to be related to the bladder or urethra, often associated with urinary urgency or frequency, in the absence of another identifiable cause. It is a diagnosis of exclusion. Current studies suggest that $2.5-6.7 \%$ of American woman suffer symptoms consistent with IC/BPS, with approximately $2.8 \%$ of urologist visits related to this disorder. It is welldocumented that IC/BPS severely impairs quality of life in patients, leading to time off of work, depression, and impairment of sexual function. Although there are several proposed theories for the etiology of IC/BPS, there is no known cause and, therefore, no known cure. In reality, this disorder is a spectrum of multiple forms of chronic pelvic pain. Treatment of these patients is challenging for several reasons. Herein, I will attempt to provide tips for managing the common challenges of treating a patient with IC/BPS.

\section{Practice tips}

\section{Tip 1: Make the most of each visit}

As mentioned in the previously featured section on evaluation, all other causes of pelvic pain must be ruled out before addressing a management plan. Patients with chronic pain conditions take a long time to accurately assess. In general, a urologist can predict this will be the case based on the referral. Therefore, it is important to book the initial time up front. Having booked the time upfront may decrease the stress of trying to "rush" these patients. Upon receiving the diagnosis of IC/BPS, patients are certain to have multiple questions.

Importantly, have the patient provide as much information prior to coming into the office or clinic room. This may include: health questionnaires, medication lists/allergies, lists of previous treatments, frequency voiding charts sequent visits, find a method to objectify the patient's symptoms in terms of the most bothersome issue and the degree of bother. It is helpful to ask:

1. What is the ONE symptom that bothers you the most? (i.e., pain, frequency, etc.)

2. On a scale of $1-10$, if 10 is ruining your life, how much does that symptom bother you?

3. If I could make ONE symptom better, what would it be?

\section{Tip 2: Set realistic expectations with the patient}

Patients with IC/BPS may experience catastrophizing behaviour, fear, and frustration. These emotions are challenging for the treating physician to deal with, particularly for busy surgeons. It is important for patients to be told that their emotional response to their symptoms is normal, or at the very least, common. Tears are normal; anger and frustration are normal. Often the anger is directed at the physician, which is further offputting to the urologist. If patients sense the frustration in the treating physician, it creates a cycle-like effect, leading to more anger in the patient.

Despite this, it is our job to set realistic expectations for these patients in order for them to learn to cope and to improve their quality of life. This includes the urologist communicating in an effective, non-abrasive fashion that there is no cure for this problem and the patient needs to learn to accept the fact that they will have to deal with this illness. Patients must be informed that there is likely not just one treatment that will alleviate all symptoms forever. In fact, treatment likely will require a multimodal approach, with effort put in on the part of the patient. There is no magic pill or surgery to entirely alleviate IC/BPS symptoms. Often, finding the best treatment for an individual will require some trial and error. The patient should know that. In order to minimize 
disappointment and further despair surrounding their diagnosis, the patient should be given a reasonable estimate of the pros, cons, and effectiveness of a chosen treatment. Patients should be given the expectation that they will have to put in effort as well, whether going to multiple physiotherapy appointments, giving up their favourite foods, or making time for stress-relieving activities like meditation or yoga.

At the end of a visit, set the expectation for how often you will be seeing the patient in followup, if at all. Let the patient know what your role will be and communicate with the referring physician regarding future tests or treatment options.

\section{Tip 3: Individualize treatment approach and multimodal therapy}

In order to find a treatment that will work, it is imperative to know what treatments have been tried and why they failed. Unfortunately, an algorithmic approach is not feasible when treating IC/BPS. As per the Canadian Urological Association guidelines, a UPOINT approach to identifying patient phenotypes may be helpful to determine which treatments may be offered to an individual (Fig. 1). One must also take into consideration: insurance coverage, allergies, other medications, side effects, patient availability, and what is available at each centre. For example, Elmiron ${ }^{\circledR}$ (pentosan polysulfate sodium) costs approximately $150-200 \$$ per 100 tablets (i.e., one month). This may not be a reasonable option due to cost. Instillation therapy may be beneficial, but if a patient has a job where they cannot make it to frequent appointments, instillations will not be feasible. It is important to keep track of what you have tried with your patient. It is also important to address bowel and sexual function in both men and women. Dysfunctional bowel symptoms and sexual dysfunction are common in patients with IC/BPS.

All patients require counselling on dietary modifications and stress-relieving measures. Pelvic floor physiotherapy may be helpful for patients, specifically if they have tenderness in the pelvic floor muscles on exam. Beyond this, it should be individualized whether or not to try more therapies initially. It depends on the severity of symptoms, possible side effects, and cost.

I generally make a point to try to entertain suggestions patients bring to my attention regarding new research and therapies for IC/BPS. I try not to brush off treatments patients may have learned about from Google or their naturopath. In a case where I cannot offer a curative therapy for patients that are so frustrated with their symptoms, I feel it is worthwhile

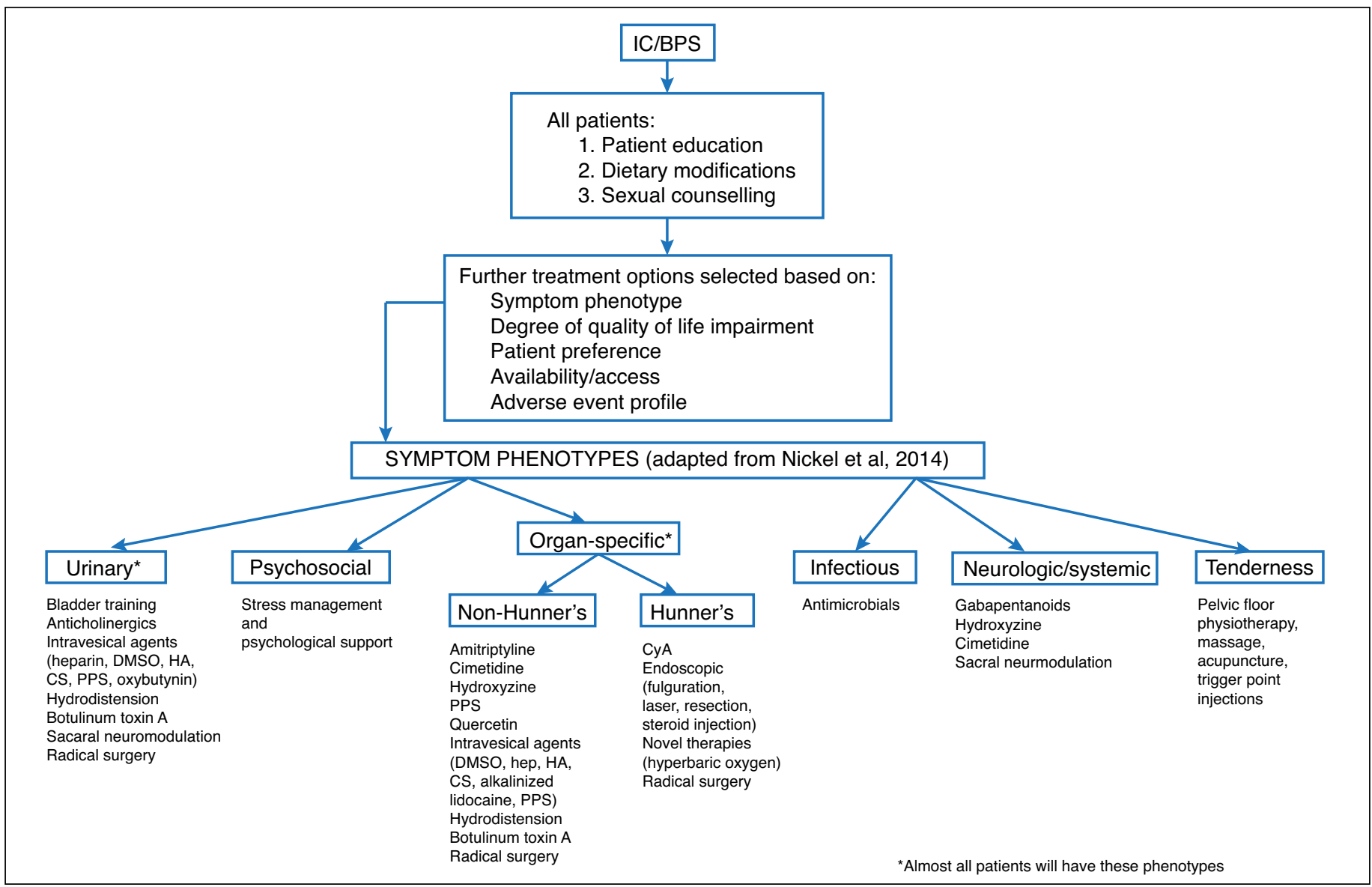

Fig. 1. Individualized approach. CS: chondroitin sulphate; DMSO: dimethyl sulfoxide; HA: hyaluronic acid; IC/BPS: interstitial cystitis/bladder pain syndrome; PPS: pentosan polysulfate sodium. 
to try to learn about the things these patients are exploring. I also state in my notes what my next plan will be if one treatment fails, which also helps to expedite future visits.

\section{Tip 4: Know your treatment options}

Like any condition in medicine, it is easier to treat if we know what to do. Tables 1 and 2 list several conservative therapies, medical options (oral, suppositories, intravesical), and surgical options for the treatment of patients with IC/ BPS. Commonly used, over-the-counter remedies include: D-mannose, Prelief ${ }^{\circledR}$, marshmallow root tea (herbal remedy), and quercetin. Baking soda (e.g., mix 1/2 tsp in one glass of water, taken orally daily) may provide some symptomatic relief. There is minimal evidence to support the use of these over-the-counter remedies. For patients with primarily urethral pain who are taking an oral contraceptive pill (OCP), I suggest a three-month trial off of the OCP. For post-menopausal woman who also have urinary tract infections, vaginal dryness, or dyspareunia, vaginal estrogen can be prescribed. Future studies are required to determine the exact role for medical marijuana in the treatment of IC/BPS. It is also helpful to be aware of any ongoing clinical trials as an option for treatment.

For patients with ulcerative IC/BPS, treatment of the lesions is of the utmost importance for improving symptoms. This may be done via fulguration, resection, or laser. Injection of steroids can be used as well. Hyperbaric oxygen is another potential option for patients (with and without ulcerative disease), where available.

It is helpful to have an approach for managing symptomatic flares, after ruling out infection. Patients again should

\begin{tabular}{|c|c|}
\hline $\begin{array}{l}\text { Conservative } \\
\text { therapies/non-medical }\end{array}$ & $\begin{array}{l}\text { - Patient education } \\
\text { - Dietary modifications } \\
\text { - Bladder retraining (urge suppression } \\
\text { techniques) } \\
\text { - Stress management } \\
\text { - Psychological support } \\
\text { - Pelvic floor physiotherapy/massage } \\
\text { - Acupuncture } \\
\text { - Hyperbaric oxygen }\end{array}$ \\
\hline $\begin{array}{l}\text { Medical therapies } \\
\text { (oral, suppositories, } \\
\text { intravesical) }\end{array}$ & - See Table 2 \\
\hline Surgical therapies & $\begin{array}{l}\text { - Hydrodistension } \\
\text { - Trigger point injections } \\
\text { - Pudendal nerve block } \\
\text { - Fulguration of Hunner's lesions } \\
\text { - Steroid injection into Hunner's lesions } \\
\text { - Botox injection } \\
\text { - Sacral neuromodulation } \\
\text { - Radical surgery (diversion/conduit) }\end{array}$ \\
\hline IC/BPS: in & pain syndrome. \\
\hline
\end{tabular}

Table 2. Medical treatment options and dosages (modified from CUA 2014 guidelines)

\begin{tabular}{|c|c|}
\hline Medication & Dosage \\
\hline Amitriptyline & 25-75 mg PO QHS \\
\hline Cimetidine & 400 mg PO BID \\
\hline Hydroxyzine & $10-50 \mathrm{mg} P O \mathrm{QHS}$ \\
\hline $\begin{array}{l}\text { Oral pentosan } \\
\text { polysulfate }\end{array}$ & 100 mg PO BID or TID \\
\hline $\begin{array}{l}\text { Intravesical } \\
\text { pentosan polysulfate }\end{array}$ & $\begin{array}{l}200 \mathrm{mg} \text { PPS mixed with } 30 \mathrm{~mL} \text { sterile } \\
\text { buffered NS retained for } 30-60 \text { minutes }\end{array}$ \\
\hline Cyclosporine A & $2-3 \mathrm{mg} / \mathrm{kg}$ divided BID \\
\hline Gabapentin & 300-2100 mg PO divided TID \\
\hline Quercetin & $500 \mathrm{mg}$ PO BID \\
\hline Pyridium & 200 mg PO TID PRN \\
\hline Buscopan & 10-20 mg PO TID PRN \\
\hline Vaginal valium supp & 10 mg PV BID PRN \\
\hline Vaginal baclofen sup & 20-30 mg PV BID PRN \\
\hline $\begin{array}{l}\text { Belladonna and } \\
\text { opiod supp }\end{array}$ & 1 supp PR q12hr PRN \\
\hline $\begin{array}{l}\text { Intravesical dimethyl } \\
\text { sulfoxide (DMSO) }\end{array}$ & $\begin{array}{l}50 \mathrm{~mL} \text { solution of } 50 \% \text { DMSO (Rimso- } 50 \text { ) } \\
\text { for } 30-60 \text { minutes, once weekly for } \\
6 \text { weeks; monthly maintenance PRN }\end{array}$ \\
\hline Intravesical heparin & $\begin{array}{c}20000-40000 \text { IU of heparin diluted in } \\
10 \mathrm{~mL} \text { NS for 30-60 minutes weekly for } \\
4-6 \text { weeks }\end{array}$ \\
\hline $\begin{array}{l}\text { Intravesical } \\
\text { hyaluronic acid }\end{array}$ & $\begin{array}{l}40 \mathrm{mg} / 50 \mathrm{~mL} \text { vial }\left(\text { Cystistat }^{\circledR}\right) \text {, weekly } \\
\text { instillations for } 4-12 \text { treatments, then } \\
\text { monthly until symptoms resolve }\end{array}$ \\
\hline $\begin{array}{l}\text { Intravesical } \\
\text { chondroitin sulfate }\end{array}$ & $\begin{array}{c}20 \mathrm{~mL} \text { vial of } 2.0 \% \text { CS (Uracyst }{ }^{\circledR} \text { ), retained } \\
30 \text { minutes weekly for } 6 \text { weeks, then } \\
\text { monthly until symptoms resolve }\end{array}$ \\
\hline $\begin{array}{l}\text { Intravesical } \\
\text { alkalinized lidocaine }\end{array}$ & $\begin{array}{c}200 \mathrm{mg} \text { lidocaine, alkalinized with a } \\
\text { sequential instillation of } 8.4 \% \mathrm{NaHCO}_{3} \\
\text { solution, to a final volume of } 10 \mathrm{~mL} \\
\text { (Urolieve }{ }^{\circledR}, \text { PSD597) }\end{array}$ \\
\hline $\begin{array}{l}\text { Intravesical } \\
\text { oxybutynin }\end{array}$ & $\begin{array}{l}10 \mathrm{mg} \text { oxybutynin (crushed tablets) diluted } \\
\text { in } 500 \mathrm{~mL} \text { NS instilled until first sensation. } \\
\text { Weekly for } 6 \text { weeks, then monthly for } \\
3 \text { months }\end{array}$ \\
\hline Hydrodistension & $\begin{array}{l}\text { Therapeutic HD under spinal or general } \\
\text { anesthesia, where the bladder is filled with } \\
\mathrm{NS} \text { by gravity drainage at a pressure of } \\
80 \mathrm{cmH}_{2} \mathrm{O} \text { to its capacity and distension } \\
\text { is maintained for } 2 \text { to no more than } 10 \\
\text { minutes. The bladder is drained at the end } \\
\text { and capacity is measured }\end{array}$ \\
\hline $\begin{array}{l}\text { Triamcinolone } \\
\text { (steroid) injection for } \\
\text { Hunner's lesions }\end{array}$ & $\begin{array}{l}1 \mathrm{~mL} \text { vial of triamcinolone }(40 \mathrm{mg} / \mathrm{mL} \text { ) } \\
\text { diluted in } 9 \mathrm{~mL} \text { NS (total } 10 \mathrm{~mL} \text { ), to be } \\
\text { injected in aliquots of } 1 \mathrm{~mL}\end{array}$ \\
\hline Botulinum toxin A & $100 \cup$ suburothelial injection \pm trigone \\
\hline
\end{tabular}

have realistic expectations that they may not be able to access their urologist any time they need. Conservative therapies, such as heat/ice packs, stress relief, and dietary changes, are important. The use of narcotics should be avoided. Medications such as pyridium, buscopan, and vaginal suppositories (e.g., valium, baclofen, gabapentin, +/-lidocaine) 
are useful on an as-needed basis. Some of these medications need to be made at a compounding pharmacy and may be costly, which is important to tell the patient. Some patients are able to learn how to do bladder instillations of heparin +/- lidocaine or Elmiron at home. In extreme cases where patients are presenting the emergency departments with painful flares, it may be helpful to have a care plan in place with the emergency department for patients to avoid unnecessary investigations, imaging, consultations to urology, and admissions to hospital.

\section{Tip 5: Refer, refer, refer}

IC/BPS is not just a urologic issue. It is likely best treated in a multidisciplinary setting, which unfortunately is hard to find. I find it very helpful to refer these patients to several other specialists who will have input into ways to improve quality of life. As mentioned in a previous section of this supplement, common referrals include: pelvic floor physiotherapy (male and female patients), gynecology and gynecological dermatology, psychologist/psychiatry, chronic pain clinic, and even gastroenterology. Unfortunately, lack of insurance/ high cost is a frequently limiting factor for patients accessing resources like pelvic floor physiotherapy and psychologists.

\section{Tip 6: Assessing outcomes}

Lastly, it is very time-consuming to reassess these patients and often challenging to determine if they have had a symptomatic improvement. By using a standard set of questioning (as described in Tip 1) or standardized questionnaires at each visit, it may be easier to judge whether or not things are going in the right directions. I often inquire, "What percentage improvement, if any, have you noticed?"

\section{Summary}

Managing patients with IC/BPS is a very challenging situation at times. All other potential causes of a patient's symptoms should be excluded, but this should not delay treatment if suspicions are high that a patient has IC/BPS. All patients should be counselled on the importance of dietary modifications and conservative therapies. Realistic patient expectations should be set early on to enhance the patient-physician relationship. As there is no cure for this poorly understood condition, many treatment options are available. An algorithmic approach to treatment of these patients may not work. Instead, an individualized, multimodal, multidisciplinary approach should be followed.

Competing interests: Dr. Cox has attended advisory boards, is a speaker for, and has received payment/honoraria from Astellas and Pfizer; and has participated in clinical trials supported by Aquinox.

This paper has been peer reviewed.

\section{Recommended reading}

1. Cox A, Golda N, Nadeau G, et al. CUA guideline: Diagnosis and treatment of interstitial cystitis/bladder pain syndrome. Can Urol Assoc J 2016;10:E136-55. https://doi.org/10.5489/cuai.3786

Correspondence: Dr. Ashley Cox, Department of Urology, Dalhousie University, Halifax, NS, Canada; ashleycox@Dal.Ca 